\title{
Development of Immune System: Conservation of Species Identity
}

\author{
Anindya Das \\ Assistant Professor, Department of Microbiology of KPC Medical College and Hospital, West \\ Bengal University of Health Science, Kolkata, India
}

\begin{abstract}
Mutual survival among different species of living organisms is quite common in our living world. That mutual survival can produce symbiotic or parasitic relationship among different living organisms. But at the same time, some relationships are harmful to the living organisms creating pathogenic relationships. Why some mutual survivals are beneficial, whereas some relationships are harmful creating different diseases in the living world? That harmful or pathological relationship producing different diseases in both the animal and plant kingdom has been extensively studied by the scientific community several times under the heading of 'Hostpathogen interaction' and 'Disease pathogenesis'. But it is still not clear why some mutual survivals are beneficial or non-harmful, whereas some co-survivals are harmful producing different disease conditions in the living world mainly due to different immune mediated reactions or direct toxic effect of substances produced by an organism. To find the answer to this question, we have to search retrospectively to the evolutionary pattern of our diverse living world. If it is assumed that we have originated from Last Universal Common Ancestor (LUCA) by different cumulative mutations, horizontal gene transfer, mobile genetic elements (MGE), transposition and natural selection, then it would be quite pragmatic to consider that two things were moving side by side in our ancient living world. On one hand it's purpose was to create the diversification of both unicellular and multi-cellular living world and on the other hand it's another purpose was to maintain the specific identity of the living organisms. It is the second purpose or the maintenance of specific identity that ultimately led to the development of Immune system.
\end{abstract}

Keywords: Pathogenic relationship, Symbiotic relationship, LUCA, Horizontal gene transfer, Recombination, Transposition, Disease pathogenesis, CRISPR-Cas \& Anti-CRISPR system.

Introduction: If we try to find the answer to the question of how the Immune System of living organisms was developed, supporting the idea of gradual evolutionary development and assuming that the diverse species forms in our living world had originated from Last Universal Common Ancestor (LUCA) [1] of unicellular organism, then we have to presume that at the early stage of evolution in the living world, diverse species of unicellular archaea, bacteria and eukaryotes were produced by cumulative mutations and different horizontal genetic material 
transfer procedures [2], recombination, mobile genetic elements (MGEs), transposition [3] and natural selection. With the help of different genetic material exchange procedures and cumulative mutations, they acquired new characteristics ultimately leading to different species form by natural selection for better survival. It can be said that cellular organisms have coevolved with various mobile genetic elements (MGEs), including transposable elements (TEs), retroelements and viruses, many of which have the capability to integrate into the host DNA. MGEs constitute $\sim 50 \%$ of mammalian genomes, $>70 \%$ of some plant genomes and up to $30 \%$ of bacterial genomes [4] (Koonin and Krupovic, 2015).

But after the development of diverse species forms in the primitive living world, though the DNA sequence is highly conserved, an innate capability to maintain the specific species form by preventing the drastic change of genetic sequence and information for that particular species probably also developed in the living world. The paradox here is that if we have to take it granted that the diverse living world has originated from last universal common ancestor (LUCA) and it had been possible by cumulative mutations and different horizontal gene transfer procedures, recombination, mobile genetic elements (MGEs), transposition [3] and natural selection, then also we have to consider that a specific tool kit of genetic information was also developed gradually to prevent diversification in living world thereby maintaining specific form. Therefore the urge to maintain the specific species form in the primitive living world probably led to the formation of specific tool kit of genetic information that ultimately shaped the structure and function of immune system in higher living organisms.

However, it is worth mentioning here that in immunoglobulin specificity, Isotype determinants refer to the genetic variations or differences in the constant region of the heavy chain of the Ig classes and subclasses within a species. But Allotype determinants refer to multiple alleles that exist for some of the genes, leading to subtle amino acid differences of Ig proteins that occur in some members of the same species and antibody to Allotype determinants can be produced by injecting antibodies from one member of a species into another member of the same species carrying different allotypic determinants. Antibody to allotypic determinants is sometimes also produced by a mother during pregnancy, in response to paternal allotypic determinants on fetal immunoglobulins and also by blood transfusion. So in higher living forms such as in mammals for example, $\mathrm{MCH}$ restriction, mismatched blood transfusion can be found as an intra-species immunological reaction or immunological reaction within different members of the same species.

Conservation of species and origin of immune system: Probably the function of the immune tool kit of genetic information in the primitive living world was to prevent the entry and assimilation of the foreign substances like DNA fragments or foreign proteins that can cause major change in the genetic information or gene regulation of a species thereby threatening their species identity. Later on in the multicellular complex living animal that toolkit in its more complex form shaped the immune system \& immune-reactive cells like lymphocytes, 
monocytes, macrophages etc. with the diversity of immunoglobulins/antibodies and TCRs generated by V(D)J recombination [5] (Kapitonov and Koonin, 2015) and during the tuning of the immune system it acquired the capability of identifying some substances as foreign to it and mounting immune response against them thereby producing host-pathogen interaction and disease pathogenesis. Though in many occasion pathogenic microorganisms have their own virulent substances and toxins with specific pharmacological action on host cell, in most of the cases immune mediated reactions are the main cause of disease manifestation.

CRISPR-Cas and anti-CRISPR system: To support this idea it is worth mentioning here that many bacteria possess a line of defense against viral infection which is a sophisticated RNAguided "immune system" called CRISPR-Cas. It provides another adaptive immune system of prokaryotic microorganisms. At the center of this system there is a surveillance complex that recognizes viral DNA and triggers its destruction. . Here, a fragment of DNA (or reversetranscribed RNA) of an infecting bacteriophage or other foreign genetic elements is integrated as spacer into a CRISPR array in the host bacterial genome [6] (Hille et al., 2018). These spacers act as immunological memory for long-term protection of the bacterial cell and the future generations also. The transcribed CRISPR array RNA (precrRNA) is later on processed into smaller crRNAs which guide sequence-specific cleavage of homologous invading nucleic acids by Cas effector nucleases. Furthermore it is also interesting to mention that, viruses can strike back and disable this surveillance complex using "anti-CRISPR" proteins. [7] [8]

Until a decade ago, scientists were not aware that bacteria had complex immune systems. But it has changed with the discovery of what is now the most famous bacterial immune mechanism: CRISPR ("clustered regularly interspaced short palindromic repeats), which is a natural gene editor. However, researchers have also identified the structure of viral anti-CRISPR proteins which attach to bacterial CRISPR surveillance complex, precisely incapacitating the bacterial defense system. The research team, co-led by biologist Gabriel C. Lander of The Scripps Research Institute (TSRI), discovered that anti-CRISPR proteins work by locking down CRISPR's ability to identify and attack the viral genome. [9] [10] [11]

It can be postulated that the CRISPR inhibitors are a natural byproduct of the evolutionary arms race between viruses and bacteria. Bacteria use CRISPR-Cas complexes to target and cut up genetic material from invading viruses. In response, viruses have developed proteins that, upon infection, can quickly bind to a host bacterium's CRISPR-Cas systems, thus nullifying their effects. In the evolutionary pathway when the CRISPR /anti-CRISPR system was developed, definitely it had some biological purposes. Assuming that the diverse species forms in our living world had originated from last universal common ancestor (LUCA), on one hand its purpose was 
to create the diversification of the unicellular living world and on the other hand its purpose was to maintain the specific identity of the living organisms.

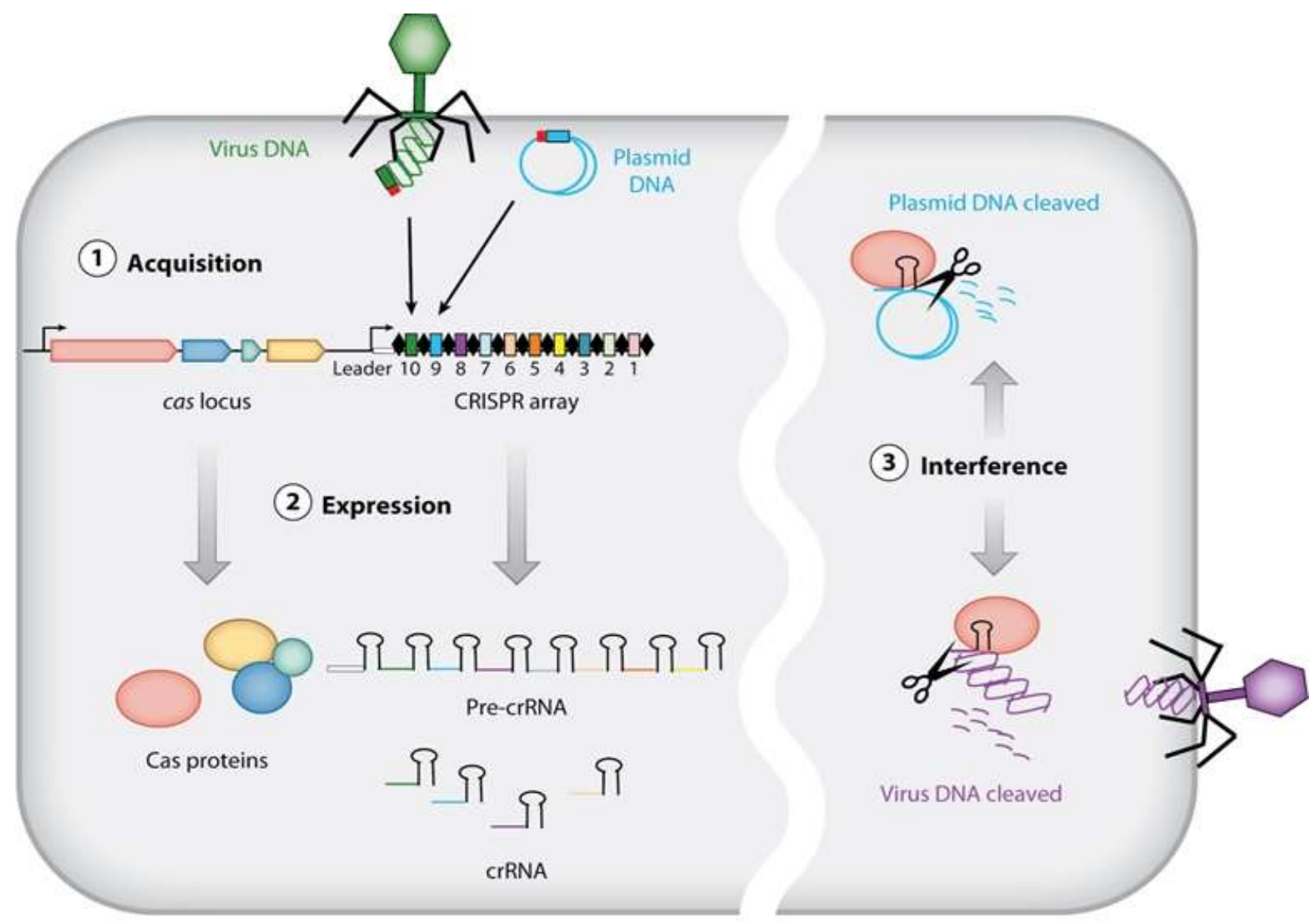

Fig 1. Schematic diagram of how the CRISPR/Cas system works (courtesy of Devaki Bhaya, Michelle Davison, and Rodolphe Barrangou originally published in Annual Review of Genetics. )

\section{Restriction Modification system (RM system) :}

Another important bacterial immune system is the restriction modification system (RM system) found in bacteria and other prokaryotic organisms, which provides a defense against foreign DNA, such as that borne by bacteriophages. With the help of restriction endonucleases, which cleave double stranded DNA, which are then further degraded by other endunucleases, bacteria prevent infection by effectively destroying foreign DNA introduced by infecting agents like bacteriophages. . Approximately one-quarter of known bacteria possess RM systems and among those bacteria about one-half have more than one type of system. 
There are four categories of restriction modification systems: type I, type II, type III and type IV, all with restriction enzyme activity and a methylase activity except for type IV that has no methylase activity. They were named in the order of discovery among them and the type II system is the most common. Restriction-modification systems are abundant in the genus Neisseria. N. meningitidis has multiple type II restriction endonuclease systems. [12] (Budroni S, et. Al. 2011) The restriction modification systems in N. meningitidis vary in specificity between different clades. Caugant and Maiden [13] (Caugant DA, Maiden MC, 2009) suggested that restriction-modification systems in meningococci may act to allow genetic exchange among very close relatives while reducing but not completely preventing genetic exchange among meningococci belonging to different clonal complexes and related species. Some viruses have evolved ways of subverting the restriction modification system, usually by modifying their own DNA, by adding methyl or glycosyl groups to it, thus blocking the restriction enzymes. Other viruses, such as bacteriophages T3 and T7, encode proteins that inhibit the restriction enzymes.

So in the evolutionary arms race between viruses and bacteria, the above observation also supports that on one hand, its purpose was to create the diversification of the unicellular living world and on the other hand it was to maintain the specific identity of the living organisms.

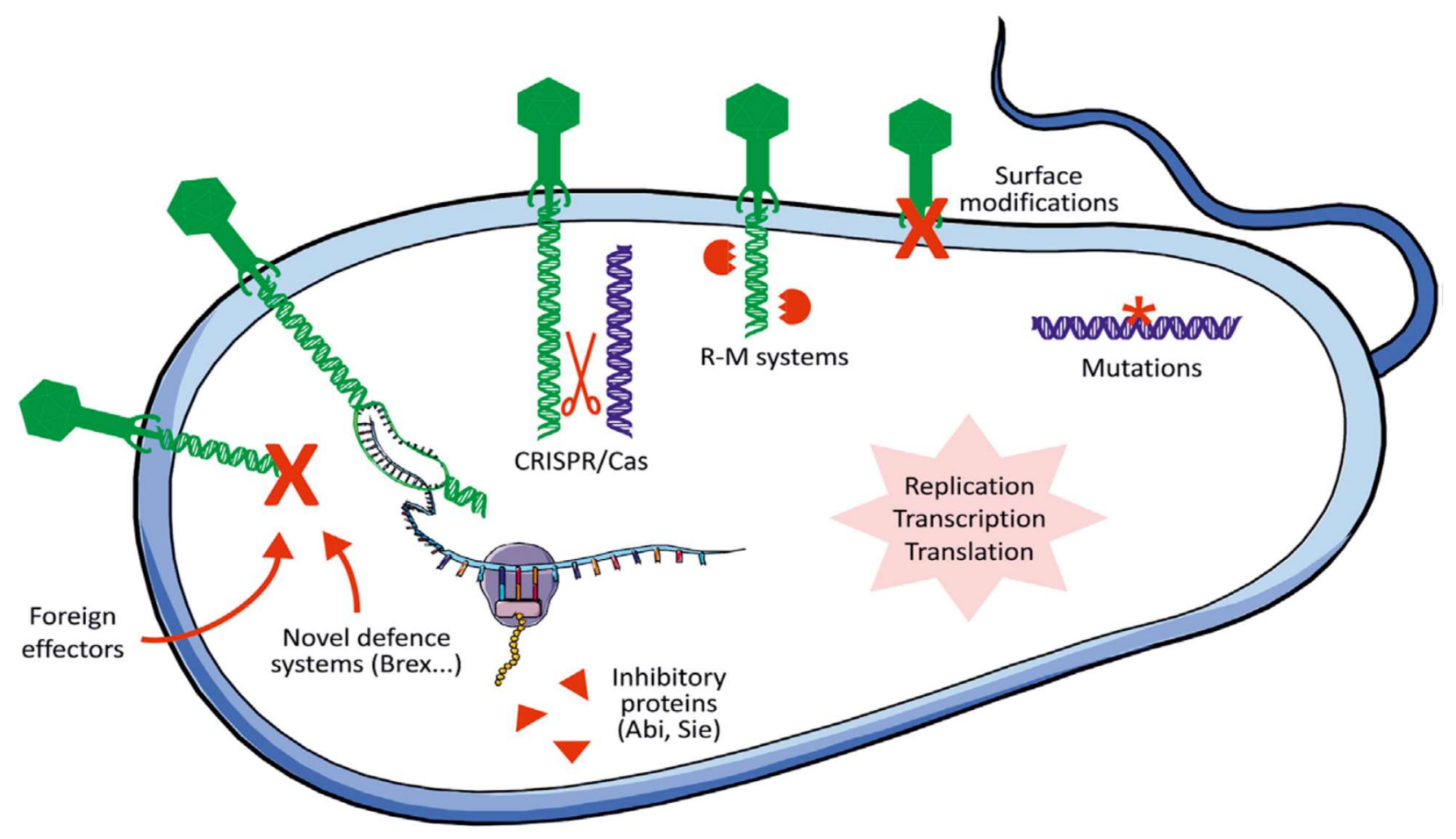

Fig 2. Bacterial Immune System ( taken from - Luisa De Sordi,Marta Lourenco and Laurent Debarbieux, The Battle Within: Interactions of Bacteriophages and Bacteria in the Gastrointestinal Tract, Cell Host \& Microbe 25, February 13, 2019) 
Superinfection exclusion (SIE) systems: At the most basic level, superinfection exclusion may be considered a prototype immune system that has been described in both prokaryotes and eukaryotes. Superinfection exclusion (SIE) systems of bacteria act in blocking invader DNA entry. These systems consist of membrane-associated proteins encoded by phages and protect the lysogenized host bacteria from infection by other closely related phages. For example, the prophage-encoded Tip protein inhibits formation of type IV pili on the surface of Pseudomonas aeruginosa [14] (Chung et al., 2014). Since these pili are common receptors for phage entry in Pseudomonas aeruginosa, Tip expression mediates Superinfection exclusion (SIE) to various phages [15] (Bondy-Denomy et al., 2016). In P. aeruginosa, three prophages are sufficient to mediate resistance against at least 30 different phages.

Prokaryotes evolve genetic material under the constant influence of, or exposure to, mobile genetic elements (MGE) such as plasmids and bacteriophages. It is quite important to note that Bacteriophages are the most abundant biological entities on earth [16] [17] (Bergh et al., 1989; Wommack and Colwell, 2000). At the same time the development of Superinfection exclusion (SIE) system in blocking invader DNA provides support in favour of development of an equally important system on the other side to maintain specific identity. Even in the ancient RNA world, a simple RNA-based immune system may have existed that had the capability of preventing superinfection with another one via trans-cleaving ribozymes.[18] (Broecker F, Moelling K, 2019)

Piwi-interacting RNA (piRNA) : They are the largest class of small noncoding RNA molecules expressed in animal cells.[19] (Seto AG et. al. 2007) piRNAs have been identified in both vertebrates and invertebrates. [20] [21] (Girard et.al. 2006 and Aravin A et. al. 2005)) They are distinct from microRNA (miRNA) in size (26-31 nucleotides as opposed to 21-24 nt), lack of sequence conservation, increased complexity, and independence of Dicer for biogenesis, at least in animals.[22] (Dorner S et. al. 2007) The wide variation in piRNA sequences and piwi function across species contributes to the difficulty in establishing the functionality of piRNAs. [23] (Wang G et. al. 2008) However, like other small RNAs, piRNAs are thought to be involved in gene silencing, specifically the silencing of transposons mainly in germline cells. [24] (Ozata DM et. al. 2019) piRNA has a role in RNA silencing via the formation of an RNA-induced silencing complex(RISC). piRNAs form RNA-protein complexes through interactions with piwi-subfamily Argonaute (Ago) proteins. piRNAs are mostly created from loci that function as transposon or jumping genes traps and provide a kind of RNA-mediated adaptive immunity against transposon expansions and invasions thereby helping in the conservation of species identity. The ability of transposons to increase genetic diversity, together with the ability of the genome to inhibit most TE activity, results in a balance that makes transposable elements an important part of evolution and gene regulation in all organisms that carry these sequences. [25] (Pray, L. 2008) 
RNA interference (RNAi) and si RNA: It is also a part of cellular immune system. It is a biological process in which RNA molecules inhibit gene expression or translation, by neutralizing targeted mRNA molecules. Andrew Fire and Craig C. Mello shared the 2006 Nobel Prize in Physiology or Medicine for their work on RNA interference in the nematode worm Caenorhabditis elegans, which they published in 1998. [26] (Daneholt B. et. al., 2007) Two types of small ribonucleic acid (RNA) molecules-microRNA (miRNA) and small interfering RNA (siRNA) - are central to RNA interference. The RNAi pathway is found in many eukaryotes, including animals, and is initiated by the enzyme Dicer, which cleaves long double-stranded RNA (dsRNA) molecules into short double-stranded fragments of 21 nucleotide siRNAs. Each siRNA is unwound into two single-stranded RNAs (ssRNAs), the passenger strand and the guide strand. The passenger strand is degraded and the guide strand is incorporated into the RNA-induced silencing complex (RISC). The most well-studied outcome is post-transcriptional gene silencing, which occurs when the guide strand pairs with a complementary sequence in a messenger RNA molecule and induces cleavage by Argonaute 2 (Ago2), the catalytic component of the RISC.[27] ( Bagasra O et. al. 2004) In plants, RNAi forms the basis of virus-induced gene silencing (VIGS), suggesting an important role in pathogen resistance. In response, many plant viruses have evolved elaborate mechanisms to suppress the RNAi response.[28] (Lucy AP et. al., 2000)

Plants mainly rely on innate immune system. It uses cell surface pattern recognition receptors (PRRs) to identify microbe associated molecular patterns (MAMPs) present in many microbes and host derived damage-associated molecular patterns (DAMPs).[29] ( Boller \& Felix 2009) Some others use disease resistance (R) proteins to respond to effector molecules secreted by pathogens.[30] (Upson et al ,2018) The perception of MAMPs or DAMPs by PRRs activates defense against invading pathogens, termed as pattern-triggered immunity (PTI) [31] (Jones \& Dangl, 2006)

RNA interference (RNAi; or RNA silencing) functions as a defense mechanism against viral infections in plants [32] (Ding, 2010). At first double-stranded RNA (dsRNA) replicative intermediates of RNA viruses are processed by Dicer like (DCL) proteins, producing viral small interfering RNAs (siRNAs). Then viral siRNAs are loaded in Argonaute (AGO) proteins to guide viral mRNA cleavage and induce RNA-based antiviral immunity [33] ( Couto \& Zipfel, 2016). Moreover, small RNAs have recently been suggested to play important roles in regulating the expression of R genes as well as PTI signaling pathways in plants [34] (Pumplin \& Voinnet, 2013).

Jawed Vertebrates (Gnathostome) Adaptive Immune System: Around 600 million years ago, multi-cellular organisms or metazoans began to appear most probably in conjunction with a dramatic increase in atmospheric oxygen levels on our planet produced by photosynthetic 
organisms. This development was followed by a remarkable diversification of metazoan species in a relatively short period of time that has been termed the "evolutionary big bang." Each metazoan lineage, including the vertebrate lineage to which we belong, appeared more than 500 million years ago.

Vertebrates with jaws (gnathostomes) possess a remarkably adaptive immune system that can recognize and initiate a protective response against potentially lethal pathogens, including bacteria, viruses, fungi, and parasites. Our adaptive immune system has an immunological memory and remembers previous pathogens encountered and can either repel a second invasion or quickly eliminates the recurrent invader by mobilizing a faster and more efficient immune response. [35] (Cooper MD, Alder MN, 2006)

Well before the evolution of an adaptive immune system, however, innate mechanisms of self-defense were acquired. Even single-cell organisms have heritable defense mechanisms, that has been discussed previously under the heading of Superinfection exclusion (SIE) systems, Restriction Modification system (RM system), CRISPR-Cas system, Piwi-interacting RNA (piRNA), RNA interference (RNAi) and si RNA and every multicellular organism appears to have a complex innate immune system [36] (Beutler, 2004).

In contrast to the adaptive immune system of prokaryotes, CRISPR-Cas, RM system, RNA interference (RNAi) and si RNA, Piwi-interacting RNA (piRNA), immunological memory in vertebrates is restricted to somatic cells and is therefore not inherited to the next generation. In jawed vertebrates, the diversity of immunoglobulins/antibodies and TCRs is generated by V(D)J recombination, in which variable (V), diversity (D) and joining (J) segments are recombined. Further antibody diversification is then achieved by somatic hypermutation [5] (Kapitonov and Koonin, 2015). Recently, it has also been observed that vertebrates without jaws (agnathans) also have an adaptive immune system and it is based on recombination and assembly of several different type of modular genetic units to generate a highly diverse repertoire of lymphocytes, each with a unique anticipatory receptor [37] [38] (Pancer et al., 2004a; Alder et al.,2005).

Conclusion: From the above discussion a picture is emerging that in unicellular bacteria the CRISPR-Cas complex, Superinfection exclusion(SIE) systems, restriction modification system (RM system) which acted as a bacterial immune system against bacteriophage attack to maintain its species identity, in higher living organisms it emerged as a complex immune system $\&$ immune-reactive cells like lymphocytes, monocytes, macrophages etc. with the diversity of immunoglobulins/antibodies and TCRs generated by V(D)J recombination, in which variable $(\mathrm{V})$, diversity (D) and joining (J) segments are recombined. If immune system would not have developed in higher living forms, then viruses and microbes after entering and multiplying in the host body and integrating their genetic material into host chromosome would have caused drastic changes in the genetic constituents of hosts forcing their species identity into crisis. The biological urge to maintain the specific form in more simple unicellular living forms probably had created a specific toolkit of genetic information that made the genetic constituents of a 
particular species highly conserved and later on shaped the immune system in higher living organisms. Some mutual survivals like Symbiosis, Mutualistic, Commensalistic, or Parasitic relations were not detrimental to their individual identity and benefitting either both species or at least one species or one species benefits while the other species is not affected and also recognized as an important selective force behind evolution, as many species have a long history of interdependent co-evolution. Whereas some relations were detrimental to their individual identity and was termed as pathogenic relationship creating a broad spectrum of host-pathogen interaction and inducing the formation of a specific toolkit of genetic information that made the genetic constituents of a particular species highly conserved and later on shaped the immune system in higher living organisms.

Acknowledgements: I personally thank all my faculty members and other staffs of the Department of Microbiology of KPC Medical College and Hospital under West Bengal University of Health Science for helping me in writing the article.

\section{References:}

1. Vaneechoutte M., Fani R. From the primordial soup to the latest universal common ancestor. Res. Microbiol. 2009;160:437-440.

2.Brown J.R. Ancient horizontal gene transfer. Nat. Rev. Genet. 2003;4:121-132.

3. Masly, John P.; Jones, Corbin D.; Mohamed, A. F. Noor; et al. (September 8, 2006). "Gene Transposition as a Cause of Hybrid Sterility in Drosophila". Science. 313 (5792): 1448-1450.

4. Koonin, E. V., and Krupovic, M. (2015). Evolution of adaptive immunity from transposable elements combined with innate immune systems. Nat. Rev. Genet. 16, 184-192.

5. Kapitonov, V. V., and Koonin, E. V. (2015). Evolution of the RAG1-RAG2 locus: both proteins came from the same transposon. Biol. Direct 10:20.

6. Hille, F., Richter, H., Wong, S. P., Bratoviè, M., Ressel, S., and Charpentier, E. (2018). The biology of CRISPR-Cas: backward and

forward. Cell 172, 1239-1259.

7. Mojica, F.J., Díez-Villaseñor, C., García-Martínez, J. et al. Intervening Sequences of Regularly Spaced Prokaryotic Repeats Derive from Foreign Genetic Elements. Journal of Molecular Evolution 60, 174-182 (2005).

8. Alexander J. Meeske,Ning Jia,Alice K. Cassel, Albina Kozlova, Jingqiu Liao, Martin Wiedmann, Dinshaw J. Patel, Luciano A. Marraffini, A phage-encoded anti-CRISPR enables 
complete evasion of type VI-A CRISPR-Cas immunity, Science 03 Jul 2020: Vol. 369, Issue 6499, pp. 54-59

9. April Pawluk, Nadia Amrani, Yan Zhang, Bianca Garcia, Yurima Hidalgo-Reyes, Jooyoung Lee, Alireza Edraki, Megha Shah, Erik J. Sontheimer, Karen L. Maxwell, Alan R.Davidson. Naturally Occurring Off-Switches for CRISPR-Cas9. Cell, 2016; DOI: 10.1016/j.cell.2016.11.017

10. Saikat Chowdhury, Joshua Carter, MaryClare F. Rollins, Sarah M. Golden, Ryan N. Jackson, Connor Hoffmann, Lyn'Al Nosaka, Joseph Bondy-Denomy, Karen L. Maxwell, Alan R. Davidson, Elizabeth R. Fischer, Gabriel C. Lander, Blake Wiedenheft. Structure Reveals Mechanisms of Viral Suppressors that Intercept a CRISPR RNA-Guided Surveillance Complex. Cell, 2017; 169 (1): 47 DOI: 10.1016/j.cell.2017.03.012

11. Seed, K.D., Lazinski, D.W., Calderwood, S.B., and Camilli, A. (2013). A bacteriophage encodes its own CRISPR/Cas adaptive response to evade host innate immunity. Nature, vol 494, issue 7438, pp 489-491; DOI: 10.1038/nature11927

12. Budroni S, Siena E, Dunning Hotopp JC, Seib KL, Serruto D, Nofroni C, Comanducci M, Riley DR, Daugherty SC, Angiuoli SV, Covacci A, Pizza M, Rappuoli R, Moxon ER, Tettelin H, Medini D (2011). "Neisseria meningitidis is structured in clades associated with restriction modification systems that modulate homologous recombination". Proc. Natl. Acad. Sci. U.S.A. 108 (11):4494 -9.

13. Caugant DA, Maiden MC (2009). "Meningococcal carriage and disease--population biology and evolution". Vaccine. 27 Suppl 2: B64-70.

14. Chung, I. Y., Jang, H. J., Bae, H. W., and Cho, Y. H. (2014). A phage protein that inhibits the bacterial ATPase required for type IV pilus assembly. Proc. Natl. Acad. Sci. U.S.A. 111, $11503-11508$.

15. Bondy-Denomy, J., Qian, J., Westra, E. R., Buckling, A., Guttman, D. S., Davidson, A. R., et al. (2016). Prophages mediate defense against phage infection through diverse mechanisms. ISME J. 10, 2854-2866.

16. Bergh,Ø, Børsheim, K. Y., Bratbak, G., and Heldal, M. (1989). High abundance of viruses found in aquatic environments. Nature 340, 467-468.

17. Wommack, K. E., and Colwell, R. R. (2000). Virioplankton: viruses in aquatic ecosystems. Microbiol. Mol. Biol. Rev. 64, 69-114. 
18.Broecker F, Moelling K. Evolution of Immune Systems From Viruses and Transposable Elements. Front Microbiol. 2019 Jan 29;10:51.

19. Seto AG, Kingston RE, Lau NC (June 2007). "The coming of age for Piwi proteins". Molecular Cell. 26 (5): 603-609.

20. Girard A, Sachidanandam R, Hannon GJ, Carmell MA (July 2006). "A germline-specific class of small RNAs binds mammalian Piwi proteins". Nature. 442 (7099): 199-202.

21. Aravin A, Tuschl T (October 2005). "Identification and characterization of small RNAs involved in RNA silencing" FEBS Letters. 579 (26): 5830-5840.

22. Dorner S, Eulalio A, Huntzinger E, Izaurralde E (August 2007). "Delving into the diversity of silencing pathways. Symposium on MicroRNAs and siRNAs: biological functions and mechanisms". EMBO Reports. 8 (8): 723-729.

23. Wang G, Reinke V (June 2008). "A C. elegans Piwi, PRG-1, regulates 21U-RNAs during spermatogenesis". Current Biology. 18 (12): 861-867.

24. Ozata DM, Gainetdinov I, Zoch A, Phillip D, Zamore PD (2019). "PIWI-interacting RNAs: small RNAs with big functions". Nature Reviews Genetics. 20 (2): 89-108.

25. Pray, L. (2008) Transposons: The jumping genes. Nature Education 1(1):204

26. Daneholt B. "Advanced Information: RNA interference". The Nobel Prize in Physiology or Medicine 2006. Archived from the original on 20 January 2007. Retrieved 25 January 2007.

27. Bagasra O, Prilliman KR (August 2004). "RNA interference: the molecular immune system". Journal of Molecular Histology. 35 (6): 545-53.

28. Lucy AP, Guo HS, Li WX, Ding SW (April 2000). "Suppression of post-transcriptional gene silencing by a plant viral protein localized in the nucleus". The EMBO Journal. 19 (7): 1672-80.

29. Boller T, Felix G. 2009. A renaissance of elicitors: perception of microbe-associated molecular patterns and danger signals by pattern-recognition receptors. Annual Review of Plant Biology 60: 379-406.

30. Upson JL, Zess EK, Białas A, Wu CH, Kamoun S. 2018. The coming of age of EvoMPMI: evolutionary molecular plant-microbe interactions across multiple timescales. Current Opinion in Plant Biology 44: 108-116. 
31. Jones JD, Dangl JL. 2006. The plant immune system. Nature 444: 323-329.

32. Ding SW. 2010. RNA-based antiviral immunity. Nature Reviews Immunology 10: 632-644.

33. Couto D, Zipfel C. 2016. Regulation of pattern recognition receptor signalling in plants. Nature Reviews Immunology 16: 537-552.

34. Pumplin N, Voinnet O. 2013. RNA silencing suppression by plant pathogens: defence, counter-defence and counter-counter-defence. Nature Reviews Microbiology 11: 745-760.

35. Cooper MD, Alder MN. The evolution of adaptive immune systems. Cell. 2006 Feb 24;124(4):815-22.

36. Beutler, B. (2004). Innate immunity: an overview. Mol. Immunol. 40, 845-859.

37. Pancer, Z., Amemiya, C.T., Ehrhardt, G.R., Ceitlin, J., Gartland, G.L., and Cooper, M.D. (2004a). Somatic diversification of variable lymphocyte receptors in the agnathan sea lamprey. Nature 430, 174-180.

38. Alder, M.N., Rogozin, I.B., Lakshminarayan, M.I., Glazko, G.V.,Cooper,M.D., and Pancer, Z. (2005). Diversity and function of adaptive immune receptors in a jawless vertebrate. Science 310, 1970-1973. 